\title{
On Thermal Dehydrochlorination of Model Compounds for PVC IV. MO Study of the Catalytic Effect of Hydrogen Chloride
}

\author{
I. TVARošKa \\ Institute of Chemistry of the Slovak Academy of Sciences, 80933 Bratislava. \\ T. BLeHA \\ Polymer Institute of the Slovak Academy of Sciences, 80934 Bratislava. \\ L. VALKO \\ Department of Physical Chemistry of the Slovak Technical University, \\ Bratislava, Czechoslovakia. \\ (Received April 27, 1974)
}

\begin{abstract}
The energy of stabilization of the transition due to the split-off molecules of $\mathrm{HCl}$ has been calculated for the elimination of $\mathrm{HCl}$ from ethyl chloride by using semiempirical methods. The analysis of the character of the energy of stabilization has shown that the electrostatic and delocalization interactions in the system are mainly responsible for the magnitude of this stabilization energy. The more polar the model of the transition state is supposed to be, the greater will be the value of the stabilization energy. Using this model, the catalytic effect of $\mathrm{HCl}$ on the destruction of poly(vinyl chloride) with an ideal structure can be described. The analogous catalytic effect on the elimination of $\mathrm{HCl}$ from defect structures of poly(vinyl chloride) is also discussed. Transition-state stabilization can explain the experimentally found decrease of activation energy of dehydrochlorination.
\end{abstract}

KEY WORDS PVC Model Compounds / Thermal Dehydrochlorination / Transition State Theory / Stabilization Energy / Frontier Molecular Orbitals / MO Theory /

One of the methods for the investigation of thermal dehydrochlorination of poly(vinyl chloride) (PVC) under very simplified conditions is the study of the destruction of various model substances which represent structural units of PVC. In the preceding paper we used the calculated activation energies of dehydrochlorination for the characterization of the thermal stability of the model substances. ${ }^{1}$ We supposed there that the elimination of hydrogen chloride proceeded by a monomolecular mechanism through a four-center cyclic transition state (TS). The mechanism of this elimination and the structure of TS were studied by semiempirical quantum-chemical methods. $^{2}$ But the relationship between the mechanism of the autocatalytic effect of $\mathrm{HCl}$ and the mechanism of PVC dehydrochlorination has not been clarified theoretically up to the present.

Originally, it was assumed ${ }^{3,4}$ that hydrogen chloride did not effect the rate of PVC dehydrochlorination. Rieche ${ }^{5}$ later pointed out that hydrogen chloride had a catalytic effect on the course of destruction in nitrogen, air, or oxygen. Talamini ${ }^{6}$ found out that the rate of PVC decomposition was 25 times greater in the presence of hydrogen chloride than it was in vacuo. Later, several authors ${ }^{7-13}$ proved that the acceleration of dehydrochlorination observed if $\mathrm{HCl}$ was present disappeared provided $\mathrm{HCl}$ was continuously removed from the system. Though these experiments have demonstrated unambigously the catalytic properties of $\mathrm{HCl}$ with respect to PVC degradation, the mechanism of this 
effect has not yet been found. Braun ${ }^{12}$ tried to explain the mechanism of the effect of $\mathrm{HCl}$ and put forward the following nonradical reaction scheme:

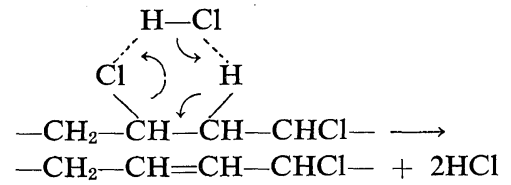

But he did not present this scheme in more detail. A radical mechanism for the effect of hydrogen chloride seems to be improbable, because the dissociation of hydrogen chloride into free readicals is unlikely below the temperature of $200^{\circ} \mathrm{C}$. The fact that the presence of $\mathrm{HCl}$ does not affect the decomposition rate of poly(vinylidene chloride), ${ }^{9,14}$ which decomposes by radical mechanism, ${ }^{15}$ also argues against a radical mechanism. Recently, an ionic mechanism for the catalytic effect of hydrogen chloride has also been suggested. ${ }^{16}$

The aim of this study is to investigate the interaction of $\mathrm{HCl}$ with the four-center cyclic transition state due to $\mathrm{HCl}$ elimination for which the parameters were evaluated in our preceding paper. $^{2}$ We assume that such an interaction stabilizes TS and gives rise to the catalytic effect of hydrogen chloride on the process of dehydrochlorination.

\section{MODEL FOR THE SUPERSYSTEM AND COMPUTATIONAL METHODS}

In order to study the mechanism of the catalytic effect of $\mathrm{HCl}$ we again used ethyl chloride as a model compound because of its simplicity and other reasons quoted in ref 2. Figure 1 shows the structure of the supersystem, i.e., TS of ethyl chloride dehydrochlorination-a molecule of $\mathrm{HCl}$. The assumed geometrical parameters are: ${ }^{2} \quad R_{1}=2.30 \AA ; \quad R_{2}=1.60 \AA ; \quad R_{3}=1.38 \AA$; $R_{4}=1.80 \mathrm{~A} ; \alpha=94.0^{\circ} ; \beta=95.0^{\circ} ; \delta=121.8^{\circ}$. The value of the dihedral angle $\gamma$ between the bonds $\mathrm{H}_{8}-\mathrm{C}_{5}-\mathrm{C}_{6}$ and $\mathrm{C}_{5}-\mathrm{C}_{6}-\mathrm{Cl}_{3}$ is equal to $90.0^{\circ}$, which means that the hydrogen atoms $\mathrm{H}_{7}, \mathrm{H}_{8}$, $\mathrm{H}_{9}$, and $\mathrm{H}_{10}$ are situated in a plane perpendicular to the plane of Figure 1. For the bond length in hydrogen chloride we used the value $r_{\mathrm{HCl}}=1.2745 \AA .^{17}$ The mutual position of both

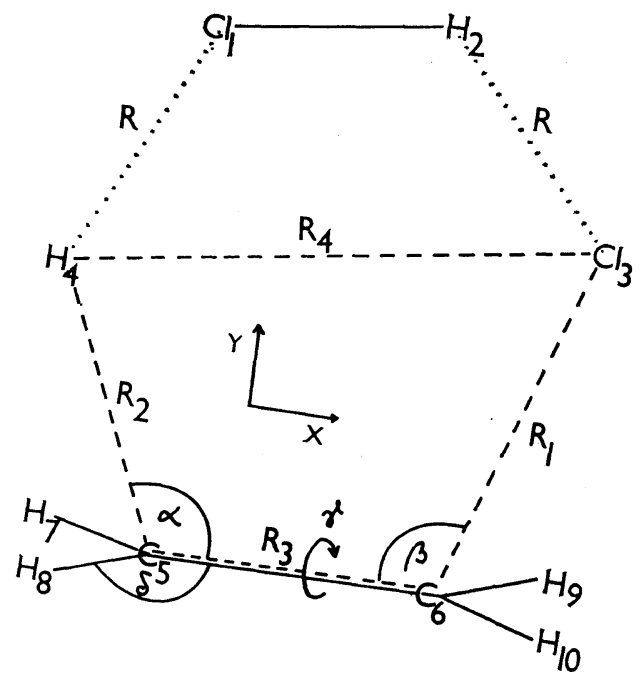

Figure 1. Geometrical structure of the interacting [TS...HCl] system.

the molecules in the course of their symmetrical approach in the plane of Figure 1 is characterized by the parameter $R$. A mutual orientation of the molecules according to Figure 1 proved to be energetically preferable. We assumed that the geometrical parameters of the system were independent of the distance between the two molecules.

The interaction energy of the supersystem [TS ...HCl] was calculated by several semiempirical quantum chemical methods in different levels of approximation. We used the extended Hückel method (EHT) and the iterative EHT with the usual parametrization, as quoted in a previous paper, ${ }^{2}$ as well as the $\mathrm{CNDO} / 2$ method with the original parametrization without including $d$-orbitals. ${ }^{18}$ Besides these quantumchemical methods we also used the partitionenergy method (PEM), in which the total interaction energy is expressed in terms of a sum of discrete contributions, in our case nonbonding van der Waals and electrostatic interactions. We considered the interatomic van der Waals repulsive and attractive pair interactions of the Buckingham type, $E_{\mathrm{vdw}}=a \exp (-b d)-$ $c d^{-6}$, where $a, b$, and $c$ were constants, $d$ was the distance between the atoms. We used the set of potential functions which proved to be convenient for the calculation of the rotation barriers of the chlorinated alkanes. ${ }^{19}$ The po- 


\section{TVarošKa, T. Bleha, and L. VAlko}

Table I. Charge distribution for individual TS models

\begin{tabular}{llcccccccc}
\hline TS & $\begin{array}{c}\text { Method } \\
\text { of } \\
\text { calculation }\end{array}$ & $\mathrm{Cl}_{1}$ & $\mathrm{H}_{2}$ & $\mathrm{Cl}_{3}$ & $\mathrm{H}_{4}$ & $\mathrm{C}_{5}$ & $\mathrm{C}_{6}$ & $\mathrm{H}_{7}, \mathrm{H}_{8}$ & $\mathrm{H}_{9}, \mathrm{H}_{10}$ \\
\hline Figure 12 & EHT & & & -0.6645 & 0.1230 & -0.1926 & 0.5085 & 0.0605 & 0.0524 \\
& IEHT & & & -0.1334 & 0.009 & -0.0272 & 0.0480 & 0.0193 & 0.366 \\
& CNDO/2a & -0.1337 & 0.1337 & -0.3863 & 0.1601 & -0.0643 & 0.1167 & 0.0382 & 0.0487 \\
& CNDO/2 & -0.1493 & 0.1497 & -0.3968 & 0.1686 & -0.0665 & 0.1190 & 0.0391 & 0.0486 \\
H-S model & EHT & & & -0.5920 & 0.1347 & -0.2635 & 0.5131 & 0.0550 & 0.0489 \\
& IEHT & & & -0.1074 & 0.0005 & -0.0453 & 0.0518 & 0.0124 & 0.0357 \\
B-H mode122 c & & & & -0.5 & 0.5 & -0.5 & 0.5 & & \\
\hline
\end{tabular}

a For isolated molecules TS and $\mathrm{HCl}$.

b For system TS... HCl in the energy minimum.

c Charges estimated by the authors of mode ${ }^{22}$.

tentials $\mathrm{H}$...H and C...C represent the modified Bartell potentials, ${ }^{20}$ while the potential according to Scott and Scheraga ${ }^{21}$ was used for the $\mathrm{Cl}$...Cl interaction. The electrostatic contribution $\left(E_{\mathrm{E} 1}\right)$ was determined from the Coulomb law in the approximation of point charges by using the value of unity for the dielectric constant.

The accuracy of the PEM method when applied to intermolecular interactions is not too great, but in spite of its simplicity this method usually gives a good picture of the characteristic changes in the investigated systems. In our case we also applied the PEM method for the comparison of the interaction energies calculated for other proposed models of the TS of $\mathrm{HCl}$ elimination. ${ }^{22,23}$ We obtained the charge distribution for individual TS models by using the EHT and IEHT methods (Table I). For the calculation of the hydrogen chloride charge we used the experimental value of the dipole moment of $\mathrm{HCl}$, namely $\mu=1.08 D^{24}$.

\section{RESULTS AND DISCUSSION}

\section{Calculation of Stabilization Energy}

The dependence on the distance $R$ of the total energy of the supersystem [TS...HCl] calculated by different methods for the geometry of Figure 1 is illustrated in Figure 2. The total energy dependence calculated by the IEHT, $\mathrm{CNDO} / 2$, and PEM methods always shows a minimum which determines the most stable configuration of the [TS...HCl] supersystem. The total energy calculated by the IEHT method

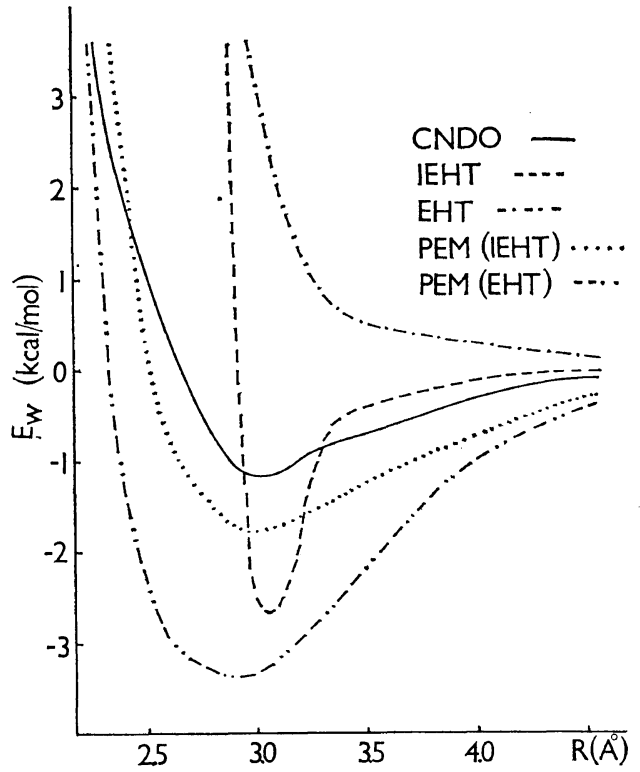

Figure 2. Variation of the total energy of the [TS...HCl] supersystem with the distance $R$ : calculated by different methods for the geometrical structure according to Figure 1. Sums of energy of isolated molecules in $\mathrm{kcal} \mathrm{mol}^{-1}$ were: $-31772.155,-11405.979$ and -10955.805 for CNDO/2, EHT, and IEHT methods, respectively.

shows an energetic minimum at the distance of $R=3.08 \AA$, the stabilization energy being $E_{\mathrm{s}}=$ $-2.68 \mathrm{kcal} \mathrm{mol}^{-1}$. According to the $\mathrm{CNDO} / 2$ calculations the minimum of total energy is at $R=3.05 \AA$, in agreement with the results of the IEHT method, but the stabilization energy is less negative $\left(E_{\mathrm{s}}=-1.18 \mathrm{kcal} \mathrm{mol}^{-1}\right)$. The total energy path calculated according to the EHT 
Effect of $\mathrm{HCl}$ on PVC Degradation by MO Theory

Table II. Stabilization energies of the TS... $\mathrm{HCl}$ system calculated for individual TS models

\begin{tabular}{llcc}
\hline Model & Method & $E_{\mathrm{s}}, \mathrm{kcal} \mathrm{mol}^{-1}$ & $R_{\min }, \AA$ \\
\hline According to Figure $1^{2}$ & EHT & - & - \\
& CNDO/2 & -1.18 & 3.05 \\
& IEHT & -2.68 & 3.08 \\
& PEM-(EHT) & -3.38 & 2.94 \\
H-S model ${ }^{23}$ & PEM-(IEHT) & & 2.94 \\
& PEM-(EHT) & -1.75 & 2.94 \\
B-H model ${ }^{22}$ & PEM-(IEHT) & -3.14 & 2.94 \\
& PEM & -1.65 & 2.94 \\
\hline
\end{tabular}

a The charges used were obtained by the EHT method.

b The charges used were obtained by the IEHT method.

method does not show any energetic minimum and the energy of the system monotonously increases if the molecules approach each other. We assume that this is due to the character of the EHT method, in which the Hamiltonian does not include any nuclear repulsion terms; it takes account of the interelectron repulsion using a very specious $H_{\mu_{\nu}}$ recipé, and it pays little attention to electron-other-nucleus attractive forces.

We used the PEM method for the same TS geometry with the two sets of charges on the individual TS atoms obtained by the EHT and IEHT methods (Table I). The minimum potential energy is at $R=2.94 \AA$ and the value of stabilization energy is $-3.38 \mathrm{kcal} \mathrm{mol} \mathrm{mon}^{-1}$ or $-1.75 \mathrm{kcal} \mathrm{mol}^{-1}$, according to the charges used.

In order to make a comparison and evaluate the effect of the geometrical and electronic TS structures on the value of the stabilization energy, we calculated by using the PEM method the interaction energy for the TS model proposed by Benson and Haugen $(\mathrm{BH})^{22}$ and the model proposed by Hasler and Setser (HS). ${ }^{23}$ The charge distribution according to the EHT and IEHT methods is given in Table I. For the $\mathrm{BH}$ model of TS we used the charges originally proposed for this model ${ }^{22}$ (Table I). The calculated stabilization energies are listed in Table II, together with the results already presented for our TS model.

\section{Analysis of Stabilization Energy from the Point of View of the PEM Method}

For the geometry of the system outlined in Figure 1 the value of the contribution of the van der Waals interactions $E_{\mathrm{vdw}}=-1.40 \mathrm{kcal}$ $\mathrm{mol}^{-1}$ ) is essentially determined by the interactions between the atoms $\mathrm{Cl}_{1} \ldots \mathrm{Cl}_{3}, \mathrm{Cl}_{1} \ldots \mathrm{H}_{4}$, and $\mathrm{H}_{2} \ldots \mathrm{Cl}_{3}$. The values of other pair interactions are approximately one order lower. In the minimum obtained by the PEM method the distance between the chlorine atoms $(d=3.36 \AA)$ is close to the value of $3.50 \AA$ which corresponds to the minimum ${ }^{19}$ of the van der Waals interaction between $\mathrm{Cl}_{1} \ldots \mathrm{Cl}_{3}$. The value of this interaction is $-0.73 \mathrm{kcal} \mathrm{mol}^{-1}$. Similarly the distance $\mathrm{H} . . \mathrm{Cl}(d=2.94 \AA)$ is not far from the value of $3.15 \AA$ corresponding to the minimum ${ }^{19}$ of the van der Waals $\mathrm{H}$...Cl interactions, whose contribution is equal to $-0.31 \mathrm{kcal} \mathrm{mol}^{-1}$. This shift of minima to smaller distances is due to the contribution of the electrostatic term $\left(E_{\mathrm{E} 1}\right)$, which in our case is always stabilizing. Its value is to a substantial degree determined by the ratio of the values of the electrostatic repulsive interactions $\left(\mathrm{Cl}_{1} \ldots \mathrm{Cl}_{3}, \mathrm{H}_{2} \ldots \mathrm{H}_{4}\right)$ and attractive interactions $\left(\mathrm{Cl}_{1} \ldots \mathrm{H}_{4}, \mathrm{H}_{2} \ldots \mathrm{Cl}_{3}\right)$. Because of the symmetrical distribution of charge in $\mathrm{HCl}$, the magnitude of the electrostatic term depends on the charge distribution in TS, in particular, on the polarity of the $\mathrm{Cl}_{3} \ldots \mathrm{H}_{4}$ bond. The electronic distribution in TS depends very much on the method used for its calculation. A higher value of the electrostatic contribution to the stabilization energy obtained by the EHT charges $\left(E_{\mathrm{E} 1}=-1.73 \mathrm{kcal} \mathrm{mol}^{-1}\right)$ when compared with the value obtained by the IEHT charges $\left(E_{\mathrm{E} 1}=-0.35 \mathrm{kcal} \mathrm{mol}^{-1}\right)$ is, therefore, quite comprehensible because it is known that the EHT method gives an exaggerated charge distribution. Similar considerations apply to the other TS models we used. 
The calculated values (Table II) of the stabilization energies increase with the polarity of the $\mathrm{Cl}_{3} \ldots \mathrm{H}_{4}$ bond, irrespective of the TS model. The magnitude of the electrostatic interaction which depends on this polarity is, therefore, an important factor for the comparison of individual TS models. For the TS model according to $\mathrm{BH}$, which shows the most polar character, we thus obtained the greatest value for the stabilization energy.

In general the PEM method seems to overestimate the stabilization energy in our case. The likely cause is the shape of the $\mathrm{Cl} \ldots \mathrm{Cl}$ potential used. A relatively high value of the attraction term of this potential may also include effectively the contribution of the electrostatic interaction in the system $\left(E_{\mathrm{E} 1}\right)$. By adding the explicitly expressed electrostatic interaction to the van der Waals interaction the same phenomenon might be evaluated twice.

\section{Analysis of Stabilization Energy from the Point} of View of the MO Method

In contrast to the PEM method, quantumchemical methods enable us to study more exactly the causes of the stabilization of the [TS... $\mathrm{HCl}]$ supersystem. For this reason, we divided, by using a $\mathrm{CNDO} / 2$ approximation, the total energy of the system into a sum of one- and two- centric terms ${ }^{18}$ to which we attributed a physical sense according to Gordon ${ }^{25}$ and Fischer and Kollmar. ${ }^{26}$

$$
E=\sum_{\mathrm{A}} E_{\mathrm{A}}+\sum_{\mathrm{A}} \sum_{\mathrm{B}} E_{\mathrm{AB}}
$$

The one-centric terms $E_{\mathrm{A}}$ express the energies of individual atoms $\mathrm{A}$. The measure of interaction between atoms $\mathrm{A}$ and $\mathrm{B}$ is given by the two-centric terms $E_{\mathrm{AB}}$ which are defined by the relationship

$$
E_{\mathrm{AB}}=E_{\mathrm{AB}}^{\mathrm{R}}+E_{\mathrm{AB}}^{\mathrm{K}}+E_{\mathrm{AB}}^{\mathrm{E}}
$$

where

$$
E_{\mathrm{AB}}^{\mathrm{R}}=2 \sum_{\mu}^{\mathrm{A}} \sum_{\nu}^{\mathrm{B}} P_{\mu_{\nu}} \beta_{\mu_{\nu}} S_{\mu_{\nu}}
$$

is a contribution of resonance integrals to the two-atom energy. A negative (positive) value of $E_{A B}^{R}$ means that the interaction between $A$ and $B$ is bonding (nonbonding). The expression

$$
K_{\mathrm{AB}}^{\mathrm{K}}=-\frac{1}{2} \gamma_{\mathrm{AB}} \stackrel{\mathrm{A}}{\Sigma}^{\mathrm{B}} \sum^{2} P_{\mu \nu}^{2}
$$

represents the stabilization caused by the exchange interactions of electrons on atoms A and $\mathbf{B}$. The expression

$$
E_{\mathrm{AB}}^{\mathrm{E}}=-P_{\mathrm{A}} Z_{\mathrm{B} \gamma_{\mathrm{AB}}}-P_{\mathrm{B}} Z_{\mathrm{A} \gamma_{\mathrm{AB}}}+P_{\mathrm{A}} P_{\mathrm{B}} \gamma_{\mathrm{AB}}+\frac{Z_{\mathrm{A}} Z_{\mathrm{B}}}{R_{\mathrm{AB}}}
$$

represents the electrostatic interaction between atoms $\mathrm{A}$ and $\mathrm{B}$. For greater internuclear distances $\gamma_{\mathrm{AB}}$ may be approximated to $1 / R_{\mathrm{AB}}$ so that $E_{\mathrm{AB}}^{\mathrm{E}}$ changes into

$$
E_{\mathbf{A} \mathrm{B}}^{\mathrm{E}}=\frac{Q_{\mathrm{A}} Q_{\mathrm{B}}}{R_{\mathrm{AB}}}
$$

All symbols in eq 1-6 are used with the usual MO theory meanings (for instance, see ref 18).

By studying the variations of the terms $\left(E_{\mathrm{A}}\right.$ and $E_{\mathrm{AB}}$ ) expressing the total energy obtained for different configurations of the [TS ... HCl] supersystem, we shall try to determine the causes of the stabilization of the system.

The stabilization energy obtained by the CNDO/2 method for the entire system of Figure 1 is equal to $-1.18 \mathrm{kcal} \mathrm{mol}^{-1}$. The most important source of stabilization energy, however, consists in the change in the sum of two-atom energies $\left(\Delta E_{\mathrm{AB}}\right)$ owing to the interaction of the atoms of $\mathrm{HCl}$ chiefly with four atoms forming a four-center TS $\left(\Delta E_{\mathrm{AB}}=-1.36 \mathrm{kcal} \mathrm{mol}^{-1}\right)$. The values of these individual terms (according to eq 2) of the two-atom energies for pair interactions as well as their resulting sums are presented in Table III. Besides this value, there are other contributions to the total stabilization energy as small variations in the energies $E_{\mathrm{AB}}$, characterizing the interactions between $\mathrm{HCl}$ and atoms $\mathrm{H}_{7}-\mathrm{H}_{10}$, as well as the differences in one-atom energies $E_{\mathrm{A}}$ for a supersystem and for isolated molecules.

The contributions of the exchange interactions $E_{\mathrm{AB}}^{\mathrm{K}}$ (Table III) to the two-atom energies are negligible in comparison with other terms. The change $\Delta E_{\mathrm{AB}}^{\mathrm{K}}$ of the exchange interactions is also very small and indicates a very small contribution from the exchange interactions to the stabilization of the supersystem.

A substantial part of the two-atom energies is represented by the term $E_{A B}^{\mathrm{E}}$, which characterizes the electrostatic Coulombic interaction (Made- 
Table III. Contributions of some two-atom interactions to the stabilization energy of the [TS...HCl] system ${ }^{a}$

\begin{tabular}{|c|c|c|c|c|}
\hline A & $E_{\mathrm{AB}}^{\mathrm{R}}$ & $E_{\mathrm{AB}}^{\mathrm{K}}$ & $E_{\mathrm{AB}}^{\mathrm{E}}$ & $E_{\mathrm{AB}}$ \\
\hline $\mathrm{Cl}_{1}-\mathrm{Cl}_{3}$ & 0.2236 & -0.0082 & 5.8683 & 6.0837 \\
\hline $\mathrm{H}$ & -0.3768 & -0.0361 & -2.6556 & -3.0685 \\
\hline $\mathrm{C}$ & 0.0267 & -0.0080 & 0.7136 & 0.7323 \\
\hline $\mathrm{C}$ & -0.0030 & -0.0084 & -1.1604 & -1.1719 \\
\hline $\mathrm{H}_{2}-\mathrm{Cl}_{3}$ & -0.5115 & -0.0319 & -6.3965 & -6.940 \\
\hline $\mathrm{H}$ & 0.0190 & -0.0007 & 2.4553 & 2.4737 \\
\hline $\mathrm{C}$ & -0.0022 & -0.0005 & -0.6626 & -0.6653 \\
\hline $\mathrm{C}$ & -0.0029 & -0.0014 & 1.1580 & 1.1538 \\
\hline$\Delta E_{\mathrm{AB}}$ & -0.63 & -0.09 & -0.68 & -1.36 \\
\hline
\end{tabular}

a $\mathrm{kcal} \mathrm{mol}^{-1}$.

lung energy) between net charges on the atoms under consideration. This is especially conspicuous in the case of the pairs of more distant atoms (Table III). The resulting contribution to the stabilization energy $\left(\Delta E_{\mathrm{AB}}^{\mathrm{E}}=-0.68 \mathrm{kcal}\right.$ $\mathrm{mol}^{-1}$ ) is no longer strong. The greater the polarity of TS and $\mathrm{HCl}$, the greater the value of this contribution. These contributions are responsible for the interaction of polar molecules at larger distance, where other terms are still very small.

If the elecrostatic term $E_{\mathrm{E} 1}$ in the PEM method is estimated by using point charges, it may be identified with the term $\Delta E_{\mathrm{AB}}^{\mathrm{E}}$. The value of $\Delta E_{A \mathrm{~B}}^{\mathrm{E}}$ obtained for all couples of atoms of the [TS... HCl] supersystem is $-0.71 \mathrm{kcal}$ $\mathrm{mol}^{-1}$ and is, therefore, situated between the values of $E_{\mathrm{E} 1}$ obtained with the EHT and IEHT charges which have been presented in the preceding part. This result is also obvious from the comparison of the charges calculated by individual methods (Table I).

The resonance contributions $E_{A B}^{\mathrm{R}}$ to two-atom energies and due to a direct interaction between the orbitals of the interacting atoms. The value of these contributions, therefore, depends on the overlap of atomic orbitals (AO) between the atoms. Though the absolute values of $E_{A B}^{R}$ in the minimum of the [TS...HCl] supersystem are lower than the $E_{\mathrm{AB}}^{\mathrm{E}}$ contributions, the contribution to the stabilization energy $\left(\Delta E_{\mathrm{AB}}^{\mathrm{R}}=\right.$ $-0.63 \mathrm{kcal} \mathrm{mol}^{-1}$ ) is almost equal to the contribution of Coulombic interactions $\Delta E_{\mathrm{AB}}^{\mathrm{E}}$. The substantial part of $\Delta E_{\mathrm{AB}}^{\mathrm{R}}$ is given by the inter- actions of the nearest atoms: i.e., $\mathrm{Cl}_{1} \ldots \mathrm{H}_{4}$, $\mathrm{H}_{2} \ldots \mathrm{Cl}_{3}$, and $\mathrm{Cl}_{1} \ldots \mathrm{Cl}_{3}$ (Table III).

On the basis of a more detail analysis of the interaction $E_{\mathrm{H}-\mathrm{Cl}}^{\mathrm{R}}$ according to eq 3 it may be stated that a substantial contribution to the bonding interaction $\mathrm{Cl}_{1}-\mathrm{H}_{4}\left(E_{\mathrm{Cl}_{1}-\mathrm{H}_{4}}^{\mathrm{R}}=-0.3768\right.$

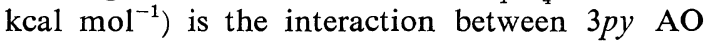
of chlorine and $1 s$ AO of hydrogen $(-0.3679$ $\left.\mathrm{kcal} \mathrm{mol}^{-1}\right)$. Similarly, in the case of the bonding interaction $\mathrm{H}_{2}-\mathrm{Cl}_{3} \quad\left(E_{\mathrm{H}_{2}}^{\mathrm{R}} \mathrm{Cl}_{3}=-0.5115 \mathrm{kcal}\right.$ $\mathrm{mol}^{-1}$ ) the most important contribution is the interaction between $3 p y \mathrm{AO}$ of chlorine and 1 s AO of hydrogen $\left(-0.3691 \mathrm{kcal} \mathrm{mol}^{-1}\right)$. The different values of both $\mathrm{H} \ldots \mathrm{Cl}$ interactions are due to a different electron density on the corresponding atoms.

On the whole, it follows from the analysis that the resonance interactions result in changetransfer and thus redistribution of the electron density in the [TS...HCl] supersystem. The charge-transfer from $\mathrm{HCl}$ to TS effects a higher polarization of the bonds in TS (Table I) as well as an increase in the electron density in the region of the $\mathrm{Cl}_{3}-\mathrm{H}_{4}$ and $\mathrm{C}_{5}-\mathrm{C}_{6}$ bonds and thus shifts the TS along the reaction coordinate nearer to the reaction products. Figure 3 provides a schematic illustration of this effect, together with the effect of electrostatic contribution. Both these contributions significantly determine the resulting stabilization energy of the system and therefore they are the main cause of the autocatalytic effect of $\mathrm{HCl}$ on dehydrochlorination.

The resonance contribution to the stabilization 
Table IV. The calculated frontier $\mathrm{MO}$ for $\mathrm{TS}$ and $\mathrm{HCl}$ and the corresponding characteristic energy values

\begin{tabular}{crl}
\hline MO & $E$, a.u. & \multicolumn{1}{c}{ Characteristic LCAO-MO coefficients } \\
\hline HOMO (TS) & -0.4070 & $0.3461\left(\mathrm{C}_{5}, 2 p y\right)+0.3538\left(\mathrm{Cl}_{3}, 3 p x\right)+0.7987\left(\mathrm{Cl}_{3}, 3 p y\right)+0.3079\left(\mathrm{H}_{4}, 1 s\right)$ \\
LUMO (TS) & -0.0210 & $0.1272\left(\mathrm{C}_{5}, 2 p y\right)+0.4969\left(\mathrm{C}_{6}, 2 p y\right)+0.3621\left(\mathrm{Cl}_{3}, 3 p x\right)+0.3819\left(\mathrm{Cl}_{3}, 3 p y\right)-0.6583\left(\mathrm{H}_{4}, 1 s\right)$ \\
HOMO (HCl) & -0.5638 & $-0.3092\left(\mathrm{Cl}_{1}, 3 p x\right)+0.9510\left(\mathrm{Cl}_{1}, 3 p y\right)$ \\
LUMO (HCl) & 0.1231 & $0.2028\left(\mathrm{Cl}_{1}, 3 s\right)+0.5954\left(\mathrm{Cl}_{1}, 3 p x\right)+0.1936\left(\mathrm{Cl}_{1}, 3 p y\right)-0.7529\left(\mathrm{H}_{2}, 1 s\right)$ \\
\hline
\end{tabular}

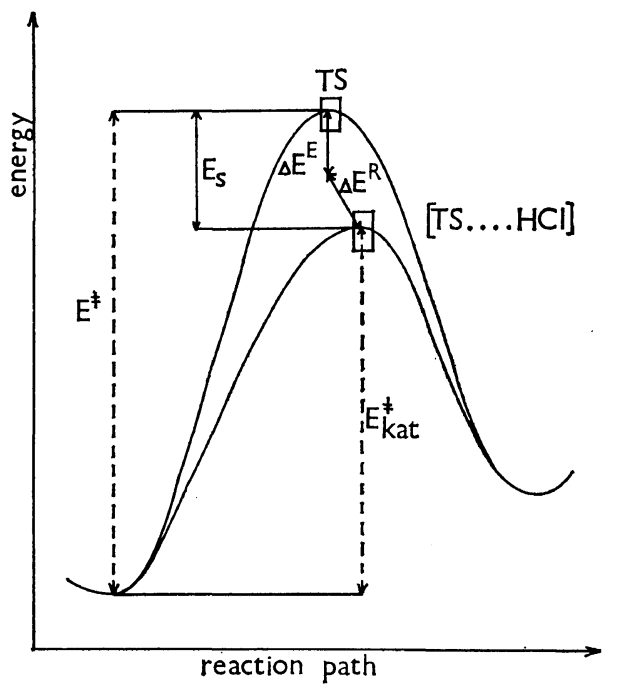

Figure 3. Schematic representation of the effect of stabilization energy and components determining this energy on the reaction pathway of dehydrochlorination.

energy may be analysed in a more telling way in terms of the pertubation theory, ${ }^{27}$ which gives a more sound idea and better understanding of the causes of charge transfer. For simplicity we shall be concerned only with the most important contributions to this interaction, i.e., with the interaction between the highest occupied (HOMO) and the lowest unoccupied molecular orbitals (LUMO) of the interacting molecules, using Fukui's frontier orbital theory. ${ }^{27}$ For this reason, it is important to know the character of HOMO and LUMO for both molecules. Because of its nature, the MO theory gives a delocalized character to each MO. But, by comparing the values of the LCAO expansion coefficients, we may determine the characteristic feature of each MO. In Table IV, the highest values of the coefficients as well as the HOMO and LUMO energies of both molecules are listed.

The nodal properties of the frontier orbitals of TS and $\mathrm{HCl}$ as well a their charge-transfer interaction are represented schematically in Figure 4. Now we shall describe Figure 4 in more detail. The HOMO of hydrogen chloride is a free electron pair of chlorine atom and LUMO is an antibonding MO with the highest frontier electron density on the $1 s$ AO of hydrogen $f_{1 s}^{\mathrm{LU}}=1.12$. The HOMO of the transition state is bonding in the region of the $\mathrm{C}_{5}-\mathrm{H}_{4}$ and $\mathrm{Cl}_{3}-\mathrm{H}_{4}$ bonds. The highest frontier electron density is concentrated on the $3 p y$ AO chlorine $\left(f_{3 p y}^{\mathrm{HO}}=1.28\right)$. The LUMO of the transition state is antibonding in the region of the $\mathrm{C}_{6}-\mathrm{Cl}_{3}$ and $\mathrm{C}_{5}-\mathrm{H}_{4}$ bonds and bonding in the region of the $\mathrm{C}_{5}-\mathrm{C}_{6}$ and $\mathrm{Cl}_{3}-\mathrm{H}_{4}$ bonds, while the highest frontier electron density is on hydrogen $\mathrm{H}_{4}$ $\left(f_{1 s}^{\mathrm{LU}}=0.88\right)$.

According to Figure 4, the most important interactions are HOMO $(\mathrm{HCl})$ and LUMO (TS) interactions and vice versa and the interactions must take place between the centers with the highest frontier electron density, i.e., the $3 p y \mathrm{AO}$ of chlorine and $1 \mathrm{~s}$ AO of hydrogen. This fact was illustrated also by the previously mentioned analysis of the resonance contribution $E_{\mathrm{H}-\mathrm{Cl}}$. The HOMO $(\mathrm{HCl})$ and LUMO (TS) interaction brings about a charge-transfer to LUMO (TS), which results in impairing the $\mathrm{C}_{6}-\mathrm{Cl}_{3}$ and $\mathrm{C}_{5}-$ $\mathrm{H}_{4}$ bonds and strengthening the $\mathrm{C}_{5}-\mathrm{C}_{6} \pi$-bond and $\mathrm{Cl}_{3}-\mathrm{H}_{4}$ bond. The transfer from HOMO (TS) to LUMO $(\mathrm{HCl})$ will impair the $\mathrm{Cl}_{1}-\mathrm{H}_{4}$ and $\mathrm{C}_{5}-\mathrm{H}_{4}$ bonds and strengthen the $\mathrm{Cl}_{3}-\mathrm{H}_{4}$. Thus the transfer results in a weakening of the cleaving bonds and a strengthening of the bonds formed in the course of the reaction. This character of electron redistribution is documented also by the change of $E_{\mathrm{AB}}$ values going from $R=\infty$ to $R=R_{\min }$. These values are -0.245 , 


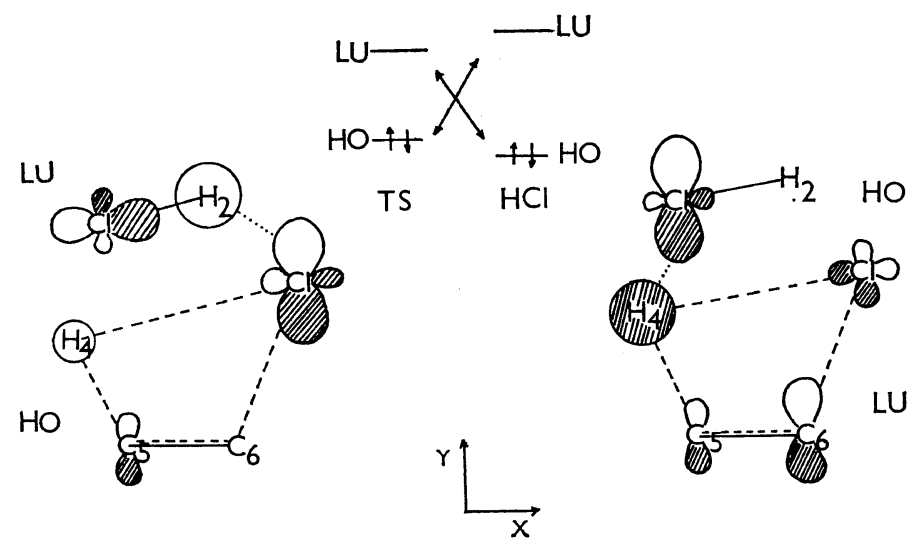

Figure 4. Schematic representation of the interaction of the frontier orbitals of TS and $\mathrm{HCl}$.

-0.342 , and $0.299 \mathrm{kcal} \mathrm{mol}^{-1}$ for $\mathrm{C}_{5}-\mathrm{H}_{4}, \mathrm{C}_{6}-$ $\mathrm{Cl}_{3}$, and $\mathrm{Cl}_{3}-\mathrm{H}_{4}$ bonds, respectively. Though the magnitude of these interactions in $\mathrm{CNDO} / 2$ approximation is small, it is in the final consequence very important for the stabilization of the $[\mathrm{TS} \ldots \mathrm{HCl}]$ supersystem.

It is worth noticing that the examination of the IEHT results by using the Mulliken population analysis ${ }^{28}$ leads to the same conclusions as the previously discussed results obtained in the CNDO/2 approximation. A similar conclusion also holds good for the choice of the geometrical structure of TS, but these effects grow with increasing polarity of the TS model.

\section{Possible Implications from the Model for PVC}

It follows unambigously from the results obtained by investigating the interactions between hydrogen chloride and TS dehydrochlorination that this interactions stabilizes TS with a simultaneous reduction of the activation energy of dehydrochlorination. From the analysis of individual contributions of stabilization energy it follows that the suggested mechanism of the catalytic effect of hydrogen chloride on dehydrochlorination is due to the Coulombic interaction of the net charges on $\mathrm{HCl}$ and TS and the direct interaction between MO of the two molecules, which results in a partial charge-transfer and depends mainly on the nodal properties of the frontier obitals.

In applying the results obtained from model calculation to PVC we suppose that dehydrochlorination occurs through a four center transi- tion state in the solid state of PVC as well. The Arhenius parameters for the unimolecular elimination reaction are influenced by the general nature of solid-phase PVC dehydrochlorination in contrast to the gas-phase low molecular compounds studies. The factors that stabilize the TS relative to the ground state, or that destabilize the ground state with respect to TS, will facilitate the unimolecular dehydrochlorination reaction and vice versa. On these grounds, we have to be careful in applying the results from low-molecular compounds to the PVC case.

The comparison of the rates for dehydrochlorination of $\mathrm{PVC}$ in the presence of $\mathrm{HCl}(v)$ with the rate when $\mathrm{HCl}$ is removed from the system $\left(v_{0}\right)$ is usually used as a test of the influence of $\mathrm{HCl}$ on PVC dehydrochlorination. Values of the increase of dehydrochlorination rate in the presence of $\mathrm{HCl}$ have been obtained over a broad interval. ${ }^{6-13}$ Considering the dehydrochlorination rate to be composed of two independent contributions, namely of noncatalyzed unimolecular elimination with rate constant $k_{0}$ as well as of autocatalyzed reaction with rate constant $k$, the rate ratio may be expressed as follows

$$
\frac{v}{v_{0}}=1+\frac{k}{k_{0}} x,
$$

where $x$ is the $\mathrm{HCl}$ amount participating in autocatalyses. From eq 7 is obvious that the increase of the dehydrochlorination rate depends 
on the increase of the rate constant $(k)$ and also on the value of $x$ which is, moreover, a function of the physical state of the degraded polymer as well as of the reaction extent. The catalytic effect of $\mathrm{HCl}$ is manifested more noticably if the concentration of this substance in the proximity of TS increases, which depends on the rate of diffusion of the liberated $\mathrm{HCl}$ in the sample. The experiments with the PVC decomposition in a solid phase have shown that the catalytic effect of $\mathrm{HCl}$ depends on the size of a particle ${ }^{10,29}$ or the critical value of the PVC film width, which is dependent on temperature. $^{29,30}$

The increase of the rate constant of autocatalytic reaction alone is caused, neglecting the change of the activation entropy, by the decrease of activation energy, which is due to the stabilizing interaction of $\mathrm{HCl}$ with TS. For the calculated values of the stabilizing energy $1.2-3.4 \mathrm{kcal} \mathrm{mol}^{-1}$ (Table II), the ratio of rate constants $k / k_{0}$ at $200^{\circ} \mathrm{C}$ is $3-40$. In the literature, two values for the decrease of activation energy of dehydrochlorination of PVC in the presence of $\mathrm{HCl}$ are given. The first value, 2 $\mathrm{kcal} \mathrm{mol}{ }^{-1},{ }^{29}$ is within the interval of the stabilization energies calculated by us, whereas the second is about triple $\left(7 \mathrm{kcal} \mathrm{mol}{ }^{-1}\right) .{ }^{11}$ This difference is most likely due to the fact that the energy of stabilization is calculated in this paper for the model of TS dehydrochlorination of ethyl chloride. Such TS for ethyl chloride may describe the dehydrochlorination from the ideal structure of PVC, whereas in the real chain various abnormal structures occur as well. The energy of stabilization of these structures will be dependent on the TS character of their dehydrochlorination. In the case of the most important abnormal structures in the chain, ${ }^{1,12,31}$ the unsaturated and branched structures may be expected; here because the mechanism of dehydrochlorination is the same as in the ideal structure, the mechanism of the autocatalytic influence of $\mathrm{HCl}$ will also be the same. On this ground we may well, even without any detailed calculation of appropriate model compounds, make a qualitative conclusion about the energy of stabilization. The ability of these structures to stabilize the cation forming on the carbon of the $\mathrm{C}-\mathrm{Cl}$ bond in TS will cause the polarity of the $\mathrm{C}-\mathrm{Cl}$ bond in $\mathrm{TS}$ to be greater in comparison with the TS of the ideal structure. For this reason, the contribution of the Coulombic interaction to the energy of stabilization will also be increased. Therefore it may be expected that the effect of $\mathrm{HCl}$ on the dehydrochlorination of these more labile structures will be still greater. The observed decrease of the activation energy, which is the mean value and dependent on the distribution of defect structures and the physical state of the polymer, will increase with increasing amount of more labil structures in the process of dehydrochlorination.

Apart from $\mathrm{HCl}$, the rate of PVC dehydrochlorination is influenced also by other compounds with mobile protons, such as organic acids, phenols, and alcohols. ${ }^{32}$ The catalytic influence of the aforementioned compounds, with respect to their structural resemblance to $\mathrm{HCl}$, will probably proceed by a mechanism similar to the one discussed in the present paper.

\section{REFERENCES}

1. L. Valko and I. Tvaroška, Eur. Polym. J., 7, 41 (1971).

2. I. Tvaroška, V. Klimo, and L. Valko, Tetrahedron, in press.

3. D. Druesedov, and C. F. Gibbs, Nat. Bul. Std. Circular, 525, 69 (1953)

4. E. J. Arlman, J. Polym. Sci., 12., 547 (1954).

5. A. Reiche, A. Grimm, and H. Mücke, Kunstoffe, 52, 265 (1962).

6. G. Talamini, G. Cinque, and G. Plama, Materie Plast Elast., 30, 317 (1964).

7. W. C. Geddes, Eur. Polym. J., 3, 267 (1967).

8. G. C. Marks, J. L. Benton, and C. M. Thomas, S.C.I. Monogr., 26, 204 (1967).

9. G. A. Razuvaev, L. S. Troitskaya, and B. B. Troitskij, J. Polym. Sci., 9, 2673 (1971).

10. M. Luther and H. Krüger, Kunststoffe, 56, 74 (1966).

11. L. S. Troitskaya, N. V. Maykov, B. B. Troitskij, and G. A. Razuvaev, Vysokomol. Soedin., Ser. $A$, 9, 2119 (1967).

12. D. Braun and R.F. Bender, Eur. Polym. J., Suppl., 269 (1969).

13. J. H. L. Henson and F. J. Hybart, J. Appl. Polym. Sci., 16, 1653 (1972).

14. J. N. Hay, J. Polym. Sci., 8, 1201 (1970). 


\section{Effect of $\mathrm{HCl}$ on PVC Degradation by MO Theory}

15. D. H. Davies, D. H. Everet, and D. J. Traylor, Trans. Faraday Soc., 67, 382 (1971).

16. S. van der Ven and V.F. de Witt, Angew. Makromol. Chem., 8, 143 (1969).

17. W. Gordy and R. L. Cook, "Microwave Molecular Spectra," Wiley-Interscience, London, 1970.

18. J. A. Pople and D. L. Beveridge, "Approximate Molecular Orbital Theory," McGrawHill, New York, N.Y, 1970.

19. A. Goursot-Leray and H. Bodot, Tetrahedron, 27, 2133 (1971).

20. L. Bartelt, J. Chem. Phys., 32, 827 (1960).

21. R. A. Scott and H. A. Scheraga, ibid., 42, 2209 (1965).

22. S. N. Benson and G. R. Haugen, J. Amer. Chem. Soc., 87, 4036 (1965).

23. J. C. Hasler and D. N. Setser, J. Chem. Phys., 45, 3246 (1966).
24. C. P. Smyth, "Dielectric Behaviour and Structure," McGraw-Hill, New York, N.Y., 1965.

25. M. S. Gordon, J. Amer. Chem. Soc., 91, 3132 (1969).

26. H. Fischer and H. Kollmar, Theor. Chim. Acta, 16, 163 (1970).

27. K. Fukui, Topics in Current Chemistry, 15, 1 (1970).

28. R. S. Mulliken, J. Chem. Phys., 23, 1833, 1841, 2338, 2343 (1955).

29. M. Carenza, Yu. V. Moiseev, and G. Palma, J. Appl. Polym. Sci., 17, 2685 (1973).

30. R. A. Pankov and V.S. Pudov, Vysokomol. Soedin., 15, 961 (1973).

31. L. Valko and I. Tvaroška, Angew. Makromol. Chem., 23, 173 (1972).

32. K. S. Minsker and G. T. Fedoseeva, "Destrukcia i stabilizacia polivinilchlorida," Chimiia, Moskva, 1972. 